Wayne State University

DigitalCommons@WayneState

Chemistry Faculty Research Publications

Chemistry

10-1-2009

\title{
Robust Quantization of a Molecular Motor Motion in a Stochastic Environment
}

\author{
Vladimir Y. Chernyak \\ WayneState University, chernyak@chem.wayne.edu \\ Nikolai A. Sinitsyn \\ Los Alamos National Laboratory, nsinitsyn@lanl.gov
}

\section{Recommended Citation}

Chernyak VY, Sinitsyn NA. (2009). Robust quantization of a molecular motor motion in a stochastic environment. J. Chem. Phys. 131, 181101 doi: $10.1063 / 1.3263821$

Available at: http://digitalcommons.wayne.edu/chemfrp/1

This Article is brought to you for free and open access by the Chemistry at DigitalCommons@WayneState. It has been accepted for inclusion in Chemistry Faculty Research Publications by an authorized administrator of DigitalCommons@WayneState. 


\title{
Robust quantization of a molecular motor motion in a stochastic environment
}

\author{
V. Y. Chernyak ${ }^{1,2}$ and N. A. Sinitsyn ${ }^{2, a)}$ \\ ${ }^{1}$ Department of Chemistry, Wayne State University, 5101 Cass Avenue, Detroit, Michigan 48202, USA \\ ${ }^{2}$ Theoretical Division, Los Alamos National Laboratory, Los Alamos, New Mexico 87545, USA
}

(Received 9 September 2009; accepted 22 October 2009; published online 10 November 2009)

\begin{abstract}
We explore quantization of the response of a molecular motor to periodic modulation of control parameters. We formulate the pumping-quantization theorem (PQT) that identifies the conditions for robust integer quantized behavior of a periodically driven molecular machine. Implication of PQT on experiments with catenane molecules are discussed. () 2009 American Institute of Physics. [doi:10.1063/1.3263821]
\end{abstract}

Molecular motors are molecules capable of performing controlled mechanical motion. An ability to rotate its parts is a crucial function of a molecular motor. ${ }^{1-3}$ It is challenging to control this motion in strongly fluctuating environment, experienced by any nanoscale system at room temperature. In the experiment, ${ }^{2}$ controlled rotation was implemented using 2- and 3-catenane molecules that are made of interlocked polymer rings ( $\mathrm{n}$-catenane is made of $\mathrm{n}$ rings). Figure 1 shows geometry of a 3-catenane molecule and its six metastable states. Small (mobile) rings perform transitions among three stations on the third, larger ring. These transitions are caused by thermal fluctuations and, alone, may not lead to directed (clockwise or counterclockwise) motion on average. In the experiments, the directed motion was induced by modulating the coupling strengths of mobile rings to stations. This forced the smaller rings to orbit around the center of the larger ring, while remaining interlocked with it.

In this communication, we address an observation ${ }^{1,2,4,5}$ that a molecular motor can perform robust quantized operations, e.g., making a full mobile ring rotation per cycle in the control parameter space, even though the ring transitions are stochastic. We illustrate the phenomenon of integer quantization using a specific example of a six-state stochastic model in Fig. 1. The problem of finding the number of rotations can be formulated in terms of stochastic single particle motion on a graph $X$ whose nodes and edges represent the metastable states and allowed transitions, respectively. The transition rates that satisfy the detailed balance can be written in Arrhenius form, i.e., they can be parameterized by the well depths $E_{i}$ and potential barriers $W_{i j}=W_{j i}$, so that the kinetic transition rate from the node $j$ to $i$ is given by $k_{i j}=e^{\beta\left(E_{j}-W_{i j}\right)}$, with $\beta=1 / k_{B} T$ being the inverse temperature. Even if the rates can be written in Arrhenius form at any time, periodic changes of well depths and barriers result in a directed particle motion. The phenomenon is referred to as the stochastic pump effect. ${ }^{4,6-8}$

For the model in Fig. 1, we further express $W_{i j}$ and $E_{i}$ in terms of the directly controllable parameters, represented by the coupling energies $\varepsilon_{j}^{i}$ of $i$ th mobile ring to $j$ th station, or the potential barriers $\omega_{n m}^{i}$ between the station $n$, occupied by the $i$ th ring, and an empty station $m$. By requesting mobile rings be unable to occupy the same station simultaneously, and by comparing state energies and kinetic rates written in different parametrizations, e.g., $E_{1}=\varepsilon_{1}^{1}+\varepsilon_{2}^{2}$ or $e^{\beta\left(E_{1}-W_{16}\right)}$ $=e^{\beta\left(\varepsilon_{1}^{1}-\omega_{13}^{1}\right)}$, we can relate the energies $\varepsilon_{j}^{i}$ and barriers $\omega_{m n}^{i}$ to $E_{k}$ and $W_{k l}$ in an effective six-state model in Fig. 1, which is, $E_{2}=\varepsilon_{1}^{1}+\varepsilon_{3}^{2}, E_{3}=\varepsilon_{2}^{1}+\varepsilon_{3}^{2}, E_{4}=\varepsilon_{2}^{1}+\varepsilon_{1}^{2}, E_{5}=\varepsilon_{3}^{1}+\varepsilon_{1}^{2}, E_{6}=\varepsilon_{3}^{1}+\varepsilon_{2}^{2}$, $W_{12}=\varepsilon_{1}^{1}+\omega_{23}^{2}, \quad W_{23}=\varepsilon_{3}^{2}+\omega_{12}^{1}, \quad W_{34}=\varepsilon_{2}^{1}+\omega_{13}^{2}, \quad W_{45}=\varepsilon_{1}^{2}+\omega_{23}^{1}$, $W_{56}=\varepsilon_{3}^{1}+\omega_{12}^{2}$, and $W_{16}=\varepsilon_{2}^{2}+\omega_{13}^{1}$.

Consider a cyclic adiabatic evolution of $\varepsilon_{1}^{1}$ and $\varepsilon_{2}^{1}$. We introduce a pump current vector $Q^{s}$, whose components $Q_{i j}^{s}$ are average numbers of times the particle passed through links $(i, j)$ during the cycle $s$ of control parameters evolution, i.e., $\boldsymbol{Q}^{s}=\int_{0}^{T} \boldsymbol{J}(t) d t$, where $\boldsymbol{J}(t)$ is the instantaneous current vector and $T$ is time of cyclic evolution of control parameters. The graph corresponding to Fig. 1 has one loop and hence $Q_{i, i+1}^{s}=Q$ for any $i$, i.e., pump currents are the same for each link.

Figure 2 shows the dependence of the number $Q$ of ring rotations per cycle on $\beta$, obtained by solving the master equation in the adiabatic limit. It shows that, generally, the system's response to a periodic parameter variation is not quantized, however, in the low-temperature $\beta \rightarrow \infty$ limit, $Q$ saturates to an integer value $Q=1$. We have checked for a number of models that the phenomenon is generic. By choosing arbitrary closed contour in the space of control parameters, followed by choosing the remaining constant parameters randomly, integer response was always achieved in the $\beta \rightarrow \infty$ limit.

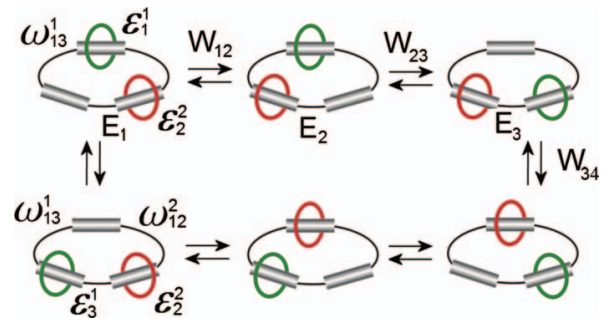

FIG. 1. Geometry and metastable states of a 3-catenane molecule.

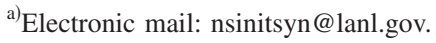




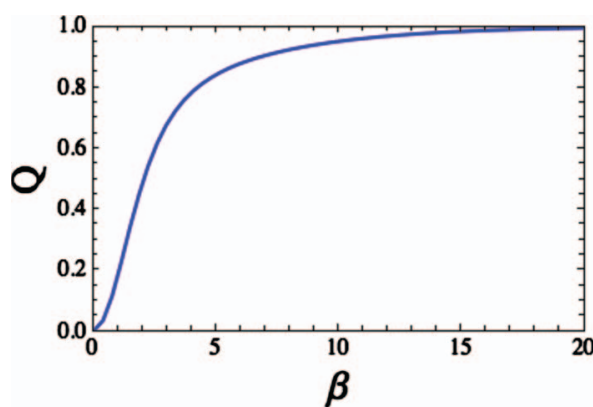

FIG. 2. Average number of rotations of mobile rings vs inverse temperature in a 3-catenane molecule after adiabatic evolution of control parameters along the contour $\epsilon_{1}^{1}=-\epsilon_{1}^{2}=\cos (\phi), \epsilon_{2}^{1}=-\epsilon_{2}^{3}=\sin (\phi)$, and $\phi \in(0,2 \pi) ; \omega_{23}^{2}$ $=1 / 4, \omega_{13}^{2}=1 / 2, \omega_{12}^{2}=-1 / 2, \omega_{12}^{1}=0, \omega_{23}^{1}=-1 / 8, \omega_{13}^{1}=-1 / 7$, and $\varepsilon_{3}^{1}=\varepsilon_{3}^{2}=0$.

The pumping-quantization theorem (PQT) rationalizes the observation and makes the following assertion: Consider any finite graph representing a Markov chain with kinetic rates written in Arrhenius form. If during the cyclic evolution of control parameters no degeneracy of the potential barriers can encounter simultaneously with degeneracy of the minimal well depths, then in the adiabatic and after this low temperature $(\beta \rightarrow \infty)$ limits, the average number of particle transitions through any link of a graph per a driving cycle is an integer.

We emphasize that by considering $\beta \rightarrow \infty$ limit after the adiabatic approximation we assume that the system has sufficient time to explore the whole phase space by making stochastic transitions before substantial change of control parameters can happen. Hence the number of rotations of the system per cycle is random and the quantization, which we discuss, appears only on average. Our arguments for the PQT are based on showing that various particle paths on a graph, which essentially contribute to the total current, are homologically equivalent to a single closed path, i.e., they differ from each other only by multiple transitions in both directions through some links. This is sufficient for a proof, since a single closed path can obviously pass through any link only an integer number of times. The proof of PQT includes three steps.

(i) First, we prove the following identity

$$
\left(\boldsymbol{J}, \boldsymbol{J}^{\mathrm{cons}}\right) \equiv \sum_{\{i, j\}} e^{\beta W_{i j}} J_{i j} J_{i j}^{\mathrm{cons}}=0,
$$

valid for the physical current $\boldsymbol{J}$ and any conserved current $\boldsymbol{J}^{\text {cons }}$ that circulates in a loop of a graph and has equal values on any link of that loop. Summation in Eq. (1) runs over the graph links. Equation (1) follows from a fact that the physical instantaneous current can always be represented in the form $J_{i j}=e^{-\beta W_{i j}}\left(e^{\beta E_{j}} \delta p_{j}-e^{\beta E_{i}} \delta p_{i}\right)$, where $\delta p_{i}$ is deviation of the probability on the $i$ th node from its equilibrium value. Taking any loop of a graph and summing $\left(e^{\beta E_{j}} \delta p_{j}-e^{\beta E_{i}} \delta p_{i}\right)$ along its links gives zero, which is equivalent to Eq. (1).

In the case of no barrier degeneracy, due to the $e^{\beta W_{i j}}$ factor in Eq. (1), the link $\{k, l\}$ with the largest barrier inside any loop on a graph dominates the scalar product (1) in the $\beta \rightarrow \infty$ limit, and the only way to make this product zero is to conclude that $\lim _{\beta \rightarrow \infty}\left(J_{k l}\right)=0$. Consider a time segment $\alpha$ without barrier degeneracies and let $Q^{\alpha}$ be the mean current integrated over the time of this segment. Integrating Eq. (1) over time leads to inequality $\left|Q_{k l}^{\alpha}\right|$ $\leq \sum_{\{i, j\} \neq\{k, l\}} \max \left(e^{\beta\left(W_{i j}-W_{k l}\right)}\right)\left|Q_{i j}^{\alpha}\right|$, where summation is over all links of the loop except the link $\{k, l\}$ with the highest barrier, and $\max (\cdots)$ means the maximum value of the expression during the given time segment. The factors $\max \left(e^{\beta\left(W_{i j}-W_{k l}\right)}\right)$ become infinitely small in the $\beta \rightarrow \infty$ limit but, for any given finite contour in the space of control parameters, $\left|Q_{i j}^{\alpha}\right|$ remains finite even in the limit of infinitely long adiabatic evolution because pump currents, on average, depend only on a choice of a path of control parameters but do not depend explicitly on time of the evolution. ${ }^{6,8}$ This leads to a stronger result $\lim _{\beta \rightarrow \infty}\left|Q_{k l}^{\alpha}\right|=0$.

(ii) Here we note that the suppression of transitions through highest loop barriers during time interval without barrier degeneracies means that complex particle motion is restricted to a subgraph $\widetilde{X}_{W} \subset X$, referred to as the maximal spanning tree, which depends only on the ordering of nondegenerate barriers $\boldsymbol{W}$. It is constructed from $X$ step-by-step by eliminating the edge with the largest barrier that does not destroy the connectivity. Eventually, when none of the links can be removed without disconnecting the graph, we obtain the tree $\widetilde{X}_{W}$. When energy $E_{j^{\prime}}$ approaches the lowest energy $E_{j}$ and then becomes a new energy minimum, the particle travels from site $j$ to site $j^{\prime}$. Let $l_{j j^{\prime}}\left(\tilde{X}_{W}\right)$ be the unique shortest path that connects $j$ to $j^{\prime}$ via $\widetilde{X}_{W}$. Although transitions form $j$ to $j^{\prime}$ are stochastic and can be done in a variety of ways, all paths from $j$ to $j^{\prime}$ on $\widetilde{X}_{W}$ are homologically equivalent to $l_{j j^{\prime}}\left(\tilde{X}_{W}\right)$.

(iii) To include events of barrier degeneracies, we partition the time period of a driving protocol into a set of small enough segments, so that for each segment we either encounter only the minimal well depth degeneracy or just the potential barrier degeneracy. We refer to them as to $0-$ and 1 -segments, respectively. If necessary, we merge the consecutive segments of the same type to make the 0 - and 1 -segments alternating. In the $\beta \rightarrow \infty$ limit, no current is generated on the 1-segment, since the populations are concentrated in the node $j$ with the lowest value of $E_{j}$ but a nonzero on average pump current is possible only when the state probability vector changes. ${ }^{8}$ The populations at the beginning and at the end of the 0 -segment $\alpha$ are concentrated, respectively, in well-defined nodes $j_{\alpha}$ and $j_{\alpha}^{\prime}$, determined by the neighboring 1-segment. Since the paths with nonvanishing probabilities from $j_{\alpha}$ to $j_{\alpha}^{\prime}$ belong to a tree $\tilde{X}_{W_{\alpha}}$, the total current $\boldsymbol{Q}^{\alpha}$, passing during the time of the 0-segment $\alpha$ has values 1 on any link on the shortest path $l_{j_{\alpha} j_{\alpha}^{\prime}}\left(\tilde{X}_{W_{\alpha}}\right)$ and it has zero values on other links. Finally, the current per cycle $Q^{s}$ $=\Sigma_{\alpha} Q^{\alpha}$ is generated by the concatenation of the consecutive paths $l_{j_{\alpha} j_{\alpha}^{\prime}}\left(\widetilde{X}_{W_{\alpha}}\right)$ that correspond to the $\alpha$-segments. This explicitly identifies the generated integer-valued current $Q^{s}$ and completes the proof of the PQT.

According to PQT, noninteger quantization in 3-catenane molecules is highly unlikely when all mobile rings and stations are different and $\beta \rightarrow \infty$. In what follows we argue that robust fractional quantization can occur in systems, where due to some symmetries, permanent (rigid) degeneracy of 


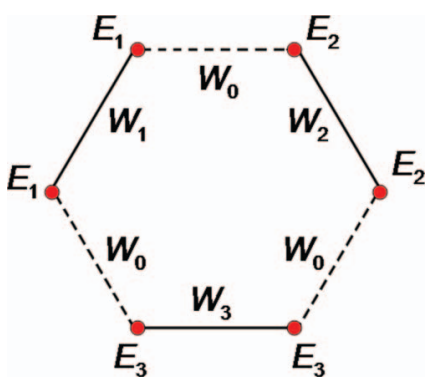

FIG. 3. The six-state model with triple degeneracy of barriers and double degeneracy of all well depths.

certain wells and/or barriers takes place. To illustrate the point we consider the 3-catenane system in Fig. 1, with a special symmetry: one of the mobile rings, called active, has three residence energies $\varepsilon_{j}$, available for control, whereas the other, passive, mobile ring has the same constant residing energy $\varepsilon=0$ for all three stations. All barriers for both rings are constant and identical, equal to $\omega$. The system is described by the Markov-chain model in Fig. 3 defined on the cyclic six-node graph $X$ with permanently degenerate wells $E_{2 j-1}=E_{2 j}=\varepsilon_{j}$, distinct barriers $W_{j}=\varepsilon_{j}+\omega$ with $j=1,2,3$, and a permanently degenerate barrier $W_{0}=\omega$. The links $\{2 j$ $-1,2 j\}$ and $\{2 j, 2 j+1\}$ describe the transitions when the passive and the active ring, respectively, switches to the nonoccupied station.

The total current $\boldsymbol{Q}^{S}$ can be calculated in a way similar to the integer-quantization case by partitioning the time period into a set of alternating 0 - and 1-segments. Consider a segment $\alpha$ with no degeneracy among the parameters $W_{k}$ with $k=0,1,2,3$. Similarly to the integer-quantized case, the largest barriers still dominate the scalar product in Eq. (1), however, they can now be degenerate. Let $\Delta p_{i}^{\alpha}$ be the change of the probability at site $i$ during the 0 -segment $\alpha$ and let $\max _{\alpha}$ be the set of links $\{i, i+1\}$ with the largest barriers during $\alpha$. Then, in the low-temperature limit, Eq. (1) combined with the continuity equations leads to

$$
\sum_{\max _{\alpha}} Q_{i, i+1}^{\alpha}=0, \quad \Delta p_{j}^{\alpha}=Q_{j-1, j}^{\alpha}-Q_{j, j+1}^{\alpha}, \quad j=1, \ldots, 5 .
$$

Equations (2) completely determine currents $\boldsymbol{Q}^{\alpha}$ on each time segment. Note that solution of Eqs. (2) results in generally rational values for $Q_{j, j+1}^{\alpha}$ because $\Delta p_{j}^{\alpha}$ take values in a set $(-1 / 2,0,1 / 2)$ and all other coefficients in equations are integers.

The protocols under consideration avoid the set $Y$ of "bad" parameters, characterized by simultaneous degeneracy of the lowest wells and highest barriers. For the model in Fig. 3, $Y$ consists of four lines $Y_{a}, a=0,1,2,3$, in the space of $\varepsilon=\left(\varepsilon_{1}, \varepsilon_{2}, \varepsilon_{3}\right)$, as it is shown in Fig. 4. To understand the global phase diagram of the quantized response, we examine the currents $Q_{a}^{s}$, the same for all links, which are generated by parameter motion along small contours enclosing $Y_{a}$ only one time. Finding the currents on all contributing 0 -segment, as described above, and then summing them yields the cur-

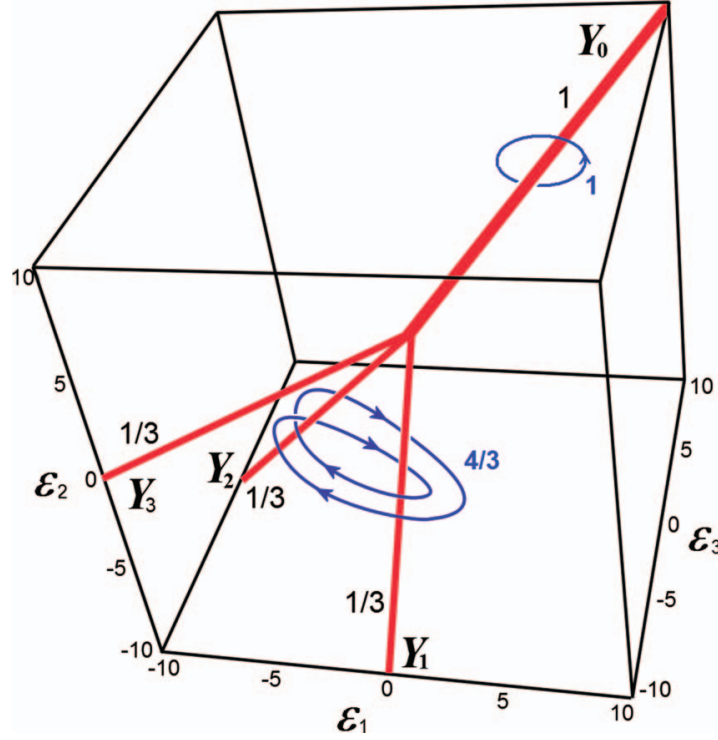

FIG. 4. Topology of the degeneracy space and currents in the active/passive ring model.

rents of 1 and 1/3, as shown in Fig. 4. The independence of $Q_{a}$ on a variation of a contour around $Y_{a}$ allows the corresponding fluxes $\mathcal{Q}_{0}=1$ and $\mathcal{Q}_{j}=1 / 3$ for $j=1,2,3$ to be associated with the lines. It also suggests a topological nature of the quantized current: the response to a general contour is given by the rational winding-index $Q^{s}=\Sigma_{a} n_{a} \mathcal{Q}_{a}$, where $n_{a}$ is the number of times the contour encloses the line $Y_{a}$, taken with a proper sign depending on orientations (see Fig. 4).

There is an obvious analogy between topological properties of pump currents and the Aharonov-Bohm effect in quantum mechanics. In the latter, the phase of the electronic wave function changes upon enclosing a quasi-onedimensional solenoid with a magnetic field by an amount proportional to the total flux of the field inside the solenoid. This analogy can be extended using the recent observation that stochastic pump effect is a geometric phenomenon ${ }^{6,8}$ in a sense that integrated over time current can be written as a contour integral in the space of control parameters $Q^{s}$ $=\oint_{s} \boldsymbol{A} \cdot d \boldsymbol{\varepsilon}$, where $\boldsymbol{A}$ is a vector potential (gauge field) in the space of control parameters. This allows an effective "magnetic field" $\boldsymbol{B}=\nabla_{\varepsilon} \times \boldsymbol{A}$ to be introduced. Our explicit calculations show that, at low temperatures, the field $\boldsymbol{B}$ is localized in narrow tubes, carrying fluxes 1 and $1 / 3$, which become $Y_{a}$-lines in the $\beta \rightarrow \infty$ limit.

The arguments leading to rational quantization in our model with degeneracies can be applied to any graph with rigid degeneracy of some barriers and/or potential wells. For example, rigid degeneracies appear in the 3-catenane model with identical mobile rings. Similar considerations predict fractional quantization with a minimal ratio $1 / 2$ for this system. We checked numerically that this fractional quantization is robust when parameters are varied keeping mobile rings identical. However, it is destroyed as soon as mobile rings are made different. 
In conclusion, we have shown that current quantization in a stochastic system is a generic phenomenon and we identified the conditions for its observation. PQT directly applies to experiments with catenane molecules predicting integerquantized response in a generic situation. We showed, however, that additional symmetries can lead to fractional quantization. For example, we predict $1 / 2$ quantization in a 3-catenane molecule that has two identical rings. We also showed that a 3-catenane molecule can demonstrate fractional quantization with a minimal ratio of $1 / 3$, which has not been observed previously. Quantization of a molecular motor response is topologically protected. This robustness should have applications to the control of nanoscale systems, experiencing thermal fluctuations.
We are grateful to M. Chertkov, J. R. Klein, and J. Horowitz for useful discussions. This material is based upon work supported by NSF under Grant Nos. CHE-0808910 and ECCS-0925618.

${ }^{1}$ D. Astumian, Proc. Natl. Acad. Sci. U.S.A. 104, 19715 (2007).

${ }^{2}$ D. A. Leigh, J. K. Y. Wong, F. Dehez, and F. Zerbetto, Nature (London) 424, 174 (2003).

${ }^{3}$ J. V. Hernández, E. R. Kay, and D. A. Leigh, Science 306, 1532 (2004); P. Hänggi and F. Marchesoni, Rev. Mod. Phys. 81, 387 (2009).

${ }^{4}$ For a review, see N. A. Sinitsyn, J. Phys. A: Math. Theor. 42, 193001 (2009).

${ }^{5}$ Y. Shi and Q. Niu, Europhys. Lett. 59, 324 (2002).

${ }^{6}$ N. A. Sinitsyn and I. Nemenman, EPL 77, 58001 (2007).

${ }^{7}$ V. Y. Chernyak and N. A. Sinitsyn, Phys. Rev. Lett. 101, 160601 (2008).

${ }^{8}$ S. Rahav, J. Horowitz, and C. Jarzynski, Phys. Rev. Lett. 101, 140602 (2008). 